\title{
Early Transthyrohyoid Injection Laryngoplasty Under Local Anaesthesia in a Single Tertiary Center of Southeast Asia: Multidimensional Voice Outcomes
}

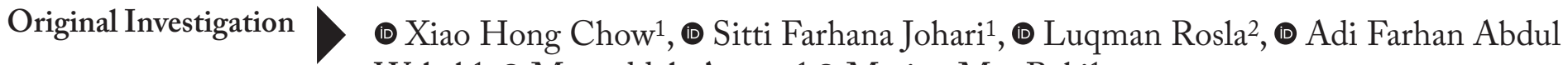 \\ Wahab ${ }^{1}$, (1) Mawaddah Azman ${ }^{1}$,® Marina Mat Baki ${ }^{1}$ \\ ${ }^{1}$ Department of Otorhinolaryngology Head and Neck Surgery, University Kebangsaan Malaysia Medical Center, \\ Kuala Lumpur, Malaysia \\ ${ }^{2}$ Department Otorhinolaryngology Head and Neck Surgery, Hospital Sultan Haji Ahmad Shah, Pahang, Malaysia
}

Abstract
ORCID ID of the authors:
X.H.C. 0000-0002-1663-6527;
S.F.J. 0000-0002-8344-4453;
L.R. 0000-0003-1585-5928;
A.F.A.W. 0000-0002-2278-9394;
M.A. 0000-0001-9138-2849;
M.M.B. 0000-0002-9282-874X.
Cite this article as: Chow XH, Johari SF, Rosla
L, Abdul Wahab AF, Azman M, Mat Baki M.
Early Transthyrohyoid Injection Laryngoplasty
Under Local Anaesthesia in a Single Tertiary
Center of Southeast Asia: Multidimensional
Voice Outcomes. Turk Arch Otorhinolaryngol
2021; 59(4): 271-81.

Objective: To study the effectiveness of early percutaneous transthyrohyoid injection laryngoplasty under local anesthesia in improving voice quality in unilateral vocal fold paralysis (UVFP) patients.

Methods: Longitudinal data of 29 UVFP patients who underwent injection laryngoplasty within six months from the onset of the symptoms were studied. The injectate $(0.5-1 \mathrm{~mL})$ was hyaluronic acid-based material (Juvéderm ULTRA XC; Allergan Industrie, France) that was delivered under local anesthesia with transthyrohyoid approach using a double-bend $21 \mathrm{G}$ needle. Multidimensional voice outcomes employing: 1) Malay-Voice-Handicap Index-10 (mVHI-10); 2) maximum phonation time (MPT); and 3) acoustic analysis [jitter\%, shimmer\% and noiseharmonic ratio (NHR)] were used to assess the treatment progress. The voice parameters were measured at baseline ( 2 weeks pre-injection), and at the first and third months post-injection.

Results: The mean age of the 29 patients was 44.69 years, with a female-to-male ratio of $3.14: 1$. The voice outcomes measured at different time points were evaluated with repeated measures ANOVA. Significant improvement was observed from baseline to three months post injection laryngoplasty for mVHI-10, jitter, and NHR ( $\mathrm{p}<0.001)$, shimmer $(\mathrm{p}=0.005)$ and MPT $(\mathrm{p}=0.018)$. Following the procedure, none of the patients developed any major complications.

Conclusion: Office setting early transthyrohyoid injection laryngoplasty using a double-bend needle is a safe and effective procedure in patients with UVFP with evidence of significant improvement in voice and life quality.

Keywords: Larynx, vocal cords, vocal cord paralysis, laryngoplasty, acoustic analysis, office surgery

Corresponding Author:

Marina Mat Baki; marinamatbaki@ppukm.ukm.edu.my

Received Date: 02.09 .2021

Accepted Date: 28.11.2021

Content of this journal is licensed under a Creative

Commons Attribution 4.0 International License. Available

online at www.turkarchotolaryngol.net

(c) (i) $\$$

D0I: 10.4274/tao.2021.2021-8-12 


\section{Introduction}

Voice plays an important role in human life. It is vital for efficient communication and expression of emotion. One of the causes of an abnormal voice is glottic insufficiency, which is commonly caused by unilateral vocal fold paralysis (UVFP). Glottic insufficiency leads to not only adverse voice quality, but also to the risk of aspiration of fluid and food material due to improper glottal closure (1). Given the considerable risks in glottic insufficiency and its adverse effects on the quality of life, early treatment is recommended.

One of the effective and less invasive modalities of early treatment for UVFP, without doubt, is injection laryngoplasty. Traditionally performed under general anesthesia, recent advancements allowed injection laryngoplasty to be performed in office settings, and thereby rendered the procedure increasingly popular in the early treatment of UVFP $(2,3)$. With the augmentation of the vocal fold by medializing the leading edge of the vocal fold, it improves the closure of the glottis (3).

Injection laryngoplasty in office settings is largely adopted via percutaneous technique which was first introduced by Ward et al. (4). One of the modifications of this technique is transthyrohyoid double-bend needle technique which was described by Achkar et al. (5). The double-bend-needle technique, a technique in which the needle was modified by creating two 45-degree angle bends with one at the needle hub and another $1-\mathrm{cm}$ proximal to the tip of the needle, allows the laryngologist to not only estimate the needle tip depth during injection, but also facilitates access to the endolarynx, which includes the entire medial and superior surface of the true and false vocal folds and the interarytenoid regions, regardless of the thyroid cartilage angulation or the chin position during the procedure (5). A study by Song et al. (6) reported that $87 \%$ of the patients who had their vocal fold augmented using this technique demonstrated subjective improvement in voice, as measured by the Voice Outcome Survey, and all patients tolerated the procedure well without complications. There are very few comprehensive studies published with multidimensional voice outcomes data on early injection laryngoplasty using the transthyrohyoid double-bend needle technique under local anesthesia.

The objective of our study was to assess the effectiveness of early injection laryngoplasty in patients with UVFP of less than 6 months, utilizing this unique double-bend needle technique under local anaesthesia. Multidimensional voice outcomes were employed to assess its efficacy in improving the voice quality objectively and subjectively.

\section{Methods}

Longitudinal data of 29 patients with UVFP who underwent injection laryngoplasty within six months from the onset of the symptoms were studied retrospectively. The cases that were included were those with UVFP of less than six months duration, who had voice issues and/or aspiration symptoms, and had undergone injection laryngoplasty under local anesthesia with temporary biomaterials. We excluded cases that had injection laryngoplasty under general anesthesia or had received long-term biomaterials, or the injection laryngoplasty was done as a touch-up procedure. Cases with incomplete data were excluded from this retrospective study.

\section{Surgical technique}

Injection laryngoplasty was performed under local anesthesia using a transthyrohyoid approach with a double-bend $21 \mathrm{G}$ needle (5). A hyaluronic based material, Juvéderm ULTRA $\mathrm{XC}$ (Allergen Industrie, France), was used as the injectate to augment the paralyzed vocal fold. The injection laryngoplasty was performed in an office setting in the clinic while the patient was sitting upright on an examination chair. First, local anesthesia was given by spraying the nostrils with Co-phenylcaine (Ent Technologies, India). Second, the subcutaneous of superior thyroid notch area were infiltrated with $1 \mathrm{cc}$ of $2 \%$ lignocaine. Third, the upper airway was anesthetized by performing intratracheal anesthesia with 3 to 4 cc of $2 \%$ lignocaine. Following this, a $21 \mathrm{G}$ needle was bent at two points as described by Achkar et al. (5). The injection was then performed under flexible nasopharyngolaryngoscope assistance, whereby the double bend-needle was introduced at the soft tissue of the superior thyroid notch and the tip of the needle appeared in the larynx below the petiole (Figure 1a). The needle was then pushed further in the midline until the distal bend was seen. Subsequently it was aimed at lateral to the vocal fold ligament in the paraglottic space, next to the vocal process (Figure 1b). Finally, the hyaluronic acid gel was slowly injected about $0.5-1 \mathrm{~mL}$ until the vocal fold was augmented with about 10\%-20\% over correction (Figure 1c).

\section{Multidimensional voice assessment}

Measurement of voice outcomes following the injection laryngoplasty was multidimensional, consisting of subjective and objective assessments. For subjective assessments, patients were evaluated with the Voice Handicap Index-10 (Bahasa Malaysia version; mVHI-10) (7). VHI-10 is a validated patient-administered subjective questionnaire that consists of physical, functional and emotional components to quantify the patient's perception of their own voice function (8). $\mathrm{mVHI}-10$ is the culturally adapted Malaysian version of VHI-10 with a total score of 40 whereby score of more than 11 is considered abnormal (7).

The objective assessments include: 1) maximum phonation time (MPT); and 2) acoustic analysis that comprises jitter $\%$ (frequency variation), shimmer \% (amplitude variation) and noise-to-harmonic ratio (NHR). The analysis of the acoustic parameters were performed using On Person Rapid 

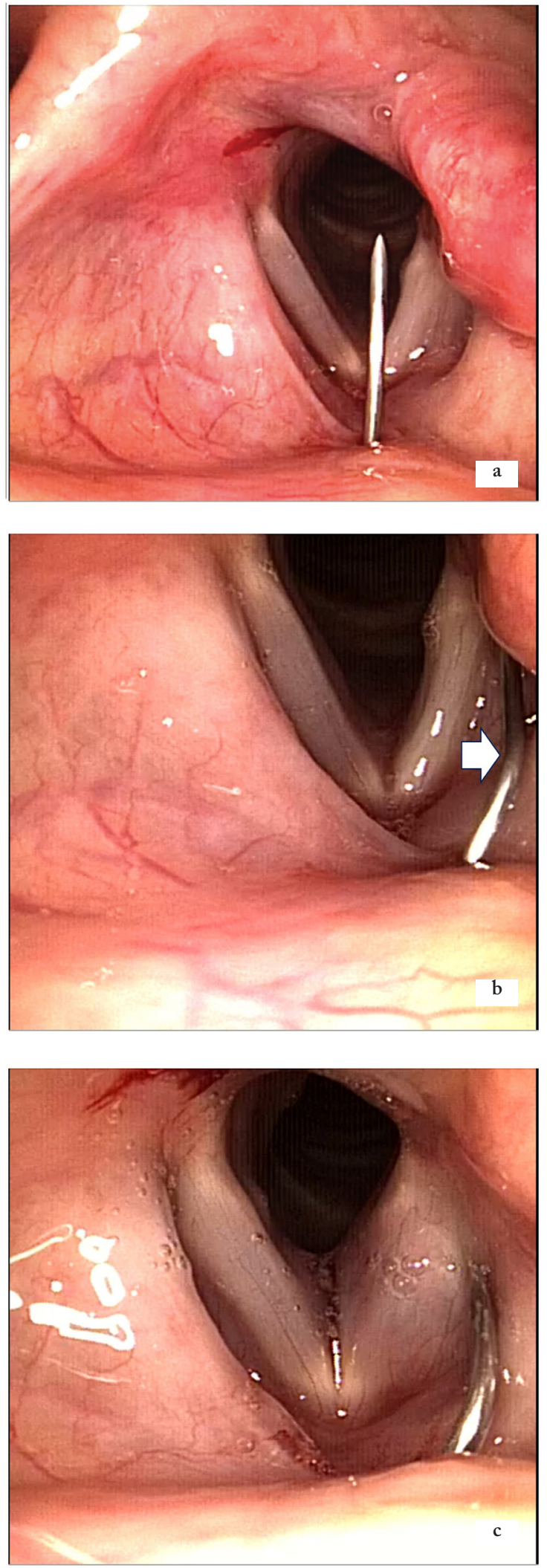

Figure 1. a) The double bend-needle enters the larynx at the petiole; b) the needle is directed to the paraglottic space. Arrowhead shows the second bend of the needle seen in the larynx; c) the paralyzed vocal fold is augmented with hyaluronic acid gel.
Voice Examiner, Oxford Research Wave Ltd (OperaVOX) in a quiet room (9). OperaVOX is objective, non-invasive, inexpensive and characterizes voice quality using intelligent tools (10). Jitter is affected by the poor control of the vocal cord vibrations with depiction of the parameter of frequency variation from cycle-to-cycle, and shimmer is affected by the glottal resistance with relation to the amplitude variation of the sound wave, while NHR assesses the components that determine speech efficacy (11). MPT, on the other hand, measures the glottic efficiency (12).

\section{Statistical Analysis}

Voice outcomes were measured at baseline, and at the first and third months post-injection. Baseline assessments were performed within the two weeks prior to the injection laryngoplasty. Multidimensional voice measurement outcomes of mVHI-10, MPT, and acoustic analysis of jitter, shimmer and NHR were analyzed with IBM SPSS Statistics version 26. Descriptive analyses of mean and standard deviation (SD) of each element were measured. Significance of the non-categorical data of multidimensional voice outcomes were analyzed with repeated measures ANOVA.

\section{Results}

In total, there were 58 UVFP patients who received temporary injection laryngoplasty (50 under local and 8 under general anesthesia). Of the 50 patients that had the procedure under local anesthesia, 21 were excluded either because of incomplete data such as incomplete documentation on the duration of UVFP or because patients did not come for the assessment of multidimensional voice outcomes. In consequence, data of 29 patients (22 females and 7 males) with a mean age of 44.69 years were reviewed. The demographic data are summarized in Table 1.

Causes of glottic insufficiency were identified as iatrogenic post thyroid surgery (72\%), idiopathic (14\%), secondary tumor $(7 \%)$, and others (7\%). The $7 \%$ other causes were secondary to old pulmonary tuberculosis and base of skull osteomyelitis. All patients underwent injection laryngoplasty under local anesthesia.

The mean and standard deviation of mVHI-10, MPT, jitter, shimmer, and NHR showed evidence of improvement from baseline pre injection laryngoplasty to months 1 and 3 post injection laryngoplasty (Table 2). Statistical analysis with repeated measures ANOVA depicted significant improvement from pre-injection to 3 months post injection laryngoplasty for mVHI-10, jitter, NHR ( $<<0.001)$, and shimmer $(\mathrm{p}=0.005)$. Patients could sustain a longer MPT in post injection laryngoplasty compared to pre injection laryngoplasty with $\mathrm{p}=0.018$. These results are presented in Table 2. 
Chow et al.

Table 1. Demographic data of patients with unilateral vocal fold paralysis

\begin{tabular}{|c|c|c|c|c|}
\hline Case & Age (years) & Gender & Etiology & Duration of paralysis (day) \\
\hline 1 & 57 & $\mathrm{~F}$ & Iatrogenic post thyroid surgery & 14 \\
\hline 2 & 38 & M & Iatrogenic post thyroid surgery & 85 \\
\hline 3 & 45 & M & Iatrogenic post thyroid surgery & 1 \\
\hline 4 & 47 & $\mathrm{~F}$ & Iatrogenic post thyroid surgery & 14 \\
\hline 5 & 67 & $\mathrm{~F}$ & Iatrogenic post thyroid surgery & 14 \\
\hline 6 & 63 & F & Iatrogenic post thyroid surgery & 14 \\
\hline 7 & 59 & $\mathrm{~F}$ & Iatrogenic post thyroid surgery & 14 \\
\hline 8 & 31 & F & Iatrogenic post thyroid surgery & 1 \\
\hline 9 & 65 & M & Idiopathic & 86 \\
\hline 10 & 31 & $\mathrm{~F}$ & Iatrogenic post thyroid surgery & 59 \\
\hline 11 & 36 & F & Base of skull osteomyelitis & 112 \\
\hline 12 & 45 & $\mathrm{~F}$ & Iatrogenic post thyroid surgery & 90 \\
\hline 13 & 34 & F & Iatrogenic post thyroid surgery & 49 \\
\hline 14 & 32 & $\mathrm{~F}$ & Secondary tumor & 6 \\
\hline 15 & 40 & $\mathrm{~F}$ & Idiopathic & 179 \\
\hline 16 & 37 & F & Iatrogenic post thyroid surgery & 90 \\
\hline 17 & 31 & M & Idiopathic & 31 \\
\hline 18 & 47 & $\mathrm{~F}$ & Iatrogenic post thyroid surgery & 110 \\
\hline 19 & 56 & M & Iatrogenic post thyroid surgery & 51 \\
\hline 20 & 48 & F & Iatrogenic post thyroid surgery & 167 \\
\hline 21 & 33 & $\mathrm{~F}$ & Iatrogenic post thyroid surgery & 115 \\
\hline 22 & 30 & F & Iatrogenic post thyroid surgery & 13 \\
\hline 23 & 61 & $\mathrm{~F}$ & Iatrogenic post thyroid surgery & 11 \\
\hline 24 & 61 & M & Iatrogenic post thyroid surgery & 116 \\
\hline 25 & 48 & M & Base of skull tumor & 3 \\
\hline 26 & 24 & $\mathrm{~F}$ & Iatrogenic post thyroid surgery & 4 \\
\hline 27 & 31 & $\mathrm{~F}$ & Idiopathic & 54 \\
\hline 28 & 31 & $\mathrm{~F}$ & Iatrogenic post thyroid surgery & 67 \\
\hline 29 & 68 & $\mathrm{~F}$ & Secondary to old pulmonary tuberculosis & 168 \\
\hline
\end{tabular}

F: Female, M: Male

Table 2. Baseline pre injection, 1 month and 3 months post injection laryngoplasty (IL) vocal function assessment

\begin{tabular}{|c|c|c|c|c|c|c|}
\hline \multirow[t]{2}{*}{ Parameter } & \multirow[t]{2}{*}{$\mathrm{n}$} & \multicolumn{3}{|c|}{$\begin{array}{l}\text { Data of vocal function assessment } \\
(\text { mean } \pm S D)\end{array}$} & \multirow{2}{*}{$\begin{array}{l}\text { Variation within } \\
\text { samples } \\
\text { F }\end{array}$} & \multirow{2}{*}{$\begin{array}{l}\text { Statistical } \\
\text { significance } \\
\text { p-value }\end{array}$} \\
\hline & & Baseline & 1-month post IL & 3-month post IL & & \\
\hline Jitter & 29 & $6.71(3.41)$ & $4.04(3.53)$ & $2.86(2.28)$ & 18.780 & 0.001 \\
\hline Shimmer & 29 & $13.37(8.41)$ & $8.47(5.32)$ & $7.02(4.21)$ & 6.459 & 0.005 \\
\hline NHR & 29 & $1.24(1.07)$ & $0.59(0.83)$ & $0.33(0.42)$ & 9.343 & 0.001 \\
\hline MPT & 29 & $5.99(4.60)$ & $7.54(4.02)$ & $9.36(4.23)$ & 4.713 & 0.018 \\
\hline mVHI-10 & 29 & $22.03(12.01)$ & $11.72(10.70)$ & $8.68(8.77)$ & 16.561 & 0.001 \\
\hline
\end{tabular}


None of the patients required nasogastric tube feeding following the injection.

\section{Discussion}

UVFP can be of various causes. In our case series, surgical iatrogenic injury represented the highest percentage in the cases of UVFP, which is in accordance with the study by Rosenthal et al. (13) who reported surgical iatrogenic injury as the most common cause with a percentage of 46.3\%. Unlike Rosenthal et al.'s (13) longitudinal study, in which non-thyroid surgeries had caused most of the surgical iatrogenic injuries, in our study the most common cause was post thyroid surgery with a rate of $72 \%$. Idiopathic and other etiologies were just at $14 \%$ and $7 \%$, respectively.

While the strong evidence of optimal management algorithm for glottic insufficiency secondary to UVFP remains a research question, injection laryngoplasty under local anaesthesia has been recommended to be a safe and effective procedure $(2,3,13)$. The aim of the procedure is to augment the flaccid vocal fold by means of injecting filler substance into the paraglottic space.

For this purpose, in recent years, a variety of injectable materials with various biocompatibility and less triggering factor of foreign body reactions have been developed (14, 15). With the development of advanced digital imaging technology, injectable material can be delivered via a fine gauge needle into the paraglottic space under direct visualization $(3,15,16)$. These advances have spurred the consideration of injection laryngoplasty as one of the effective treatments for glottic insufficiency $(17,18)$. In office setting, injection laryngoplasty is generally performed via percutaneous approaches which can be subdivided into transthyroid, transthyrohyoid and transcricothyroid (19).

Avoidance of general anesthesia is the most favorable aspect of injection laryngoplasty done in an office setting. With the patient awake and seated upright, injection laryngoplasty under local anesthesia allows real-time phonation monitoring together with the titration of the injected material amount, which is not possible under general anesthesia (3). Measurement of the voice and the airway status with the rate of permeability of the injected material location can be assessed simultaneously during injection laryngoplasty under local anesthesia (19).

Office setting injection laryngoplasty is particularly advantageous for patients with multiple comorbidities and high anesthetic risk, as it reduces perioperative morbidity with shortened hospital stay $(15,17)$. Notably, anticoagulation is not a contraindication as the risk of bleeding and hematoma formation is low for injection laryngoplasty under local anesthesia (18). Office setting injection laryngoplasty is more cost-effective compared to general anesthesia, and thereby more affordable and accessible for the general population. Moreover, it helps to reduce the burden of the already rising healthcare cost to the country $(3,15)$. An added advantage of injection laryngoplasty under local anesthesia is the possibility of avoiding the long waiting time for an operation theater (OT) slot, which can in turn be reserved for more urgent, i.e., malignant cases that require longer OT times.

Prediction of the prognosis of UVFP is uncompromising as the best timing for performing injection laryngoplasty has been controversial previously, and there has been rationale on a waiting period of 6 to 12 months prior to intervention in certain selected cases of UVFP for possible spontaneous recovery occurrence. Recent research advocated early injection laryngoplasty in UVFP as it has been reported to statistically reduce the need for future open laryngeal framework surgery (19-21). A study by Choi et al. (22) showed that early injection laryngoplasty within one month following iatrogenic UVFP significantly improved the voice outcomes with reduction of aspiration risk.

With the convenience of the facilities in the office setting and its low complication rate, this concept provides an excellent early therapeutic option for patients with UVFP. Comprehensive clinical measurement of the voice outcome post injection laryngoplasty is essential.

A retrospective study by Bové et al. (3) comparing the clinical efficacy of injection laryngoplasty under general and local anesthesia revealed that both outcomes demonstrated similar promising improvement of voice quality based on VHI-10, thus advocated awake injection laryngoplasty in prudent patients. Powell et al. (14) assessed the subjective clinical outcome of injection laryngoplasty under local anesthesia with hyaluronic acid and calcium hydroxylapatite in 68 subjects using Voice Performance Questionnaires and GRBAS, whereas a study by Mohammed et al. (17) studied the clinical outcome of injection laryngoplasty with calcium hydroxylapatite in outpatient setting in 21 subjects solely with VHI-10. Singh and Gupta (17) evaluated videostroboscopic findings, acoustic analysis and VHI post injection laryngoplasty with calcium hydroxylapatite in only 12 patients. All three studies demonstrated relative significant improvement on the voice outcome post injection laryngoplasty under local anesthesia. Table 3 shows a comprehensive comparison of the number of patients, approaches adopted, materials used, measurement of voice outcomes and complications of injection laryngoplasty under local anesthesia in various studies. 


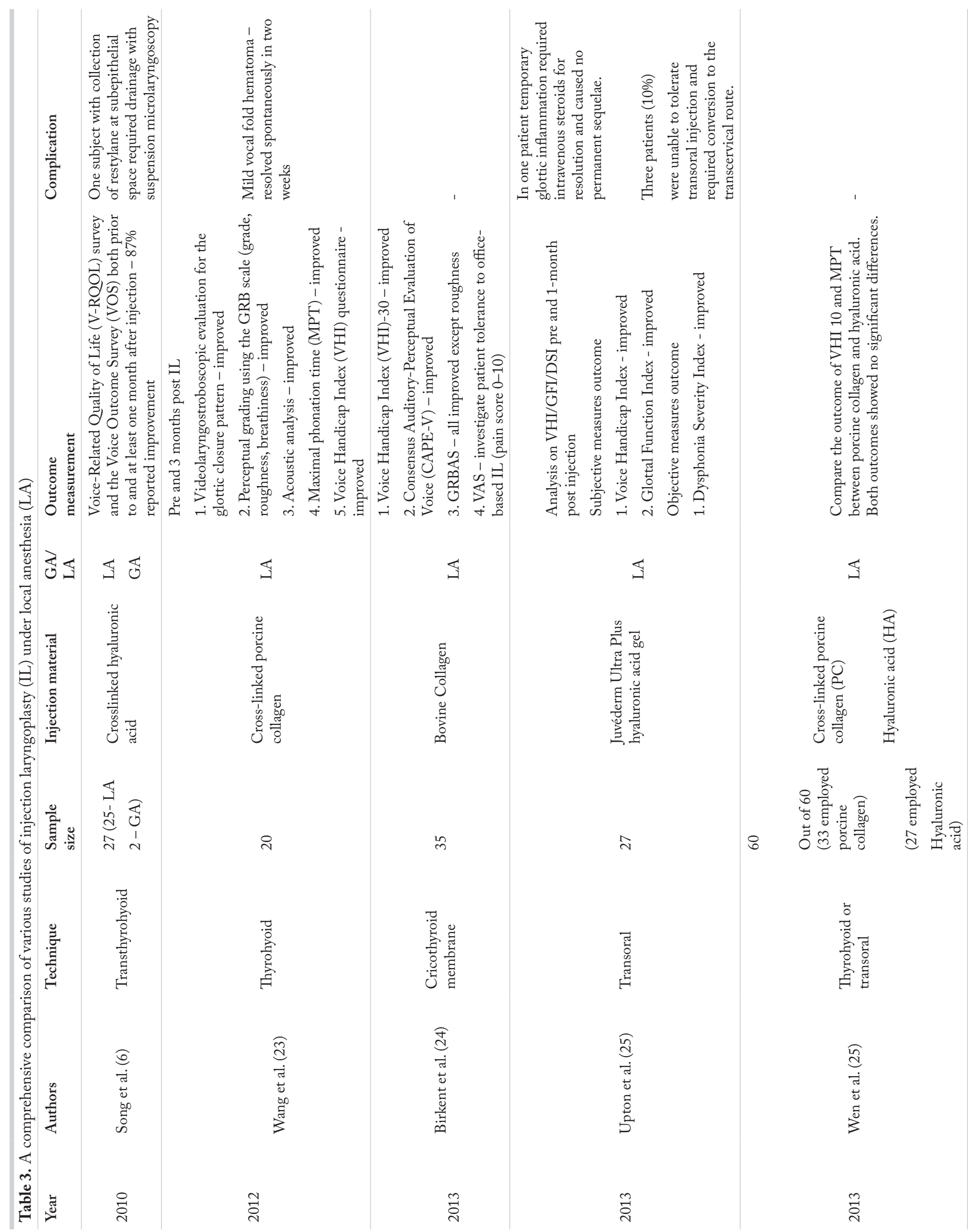




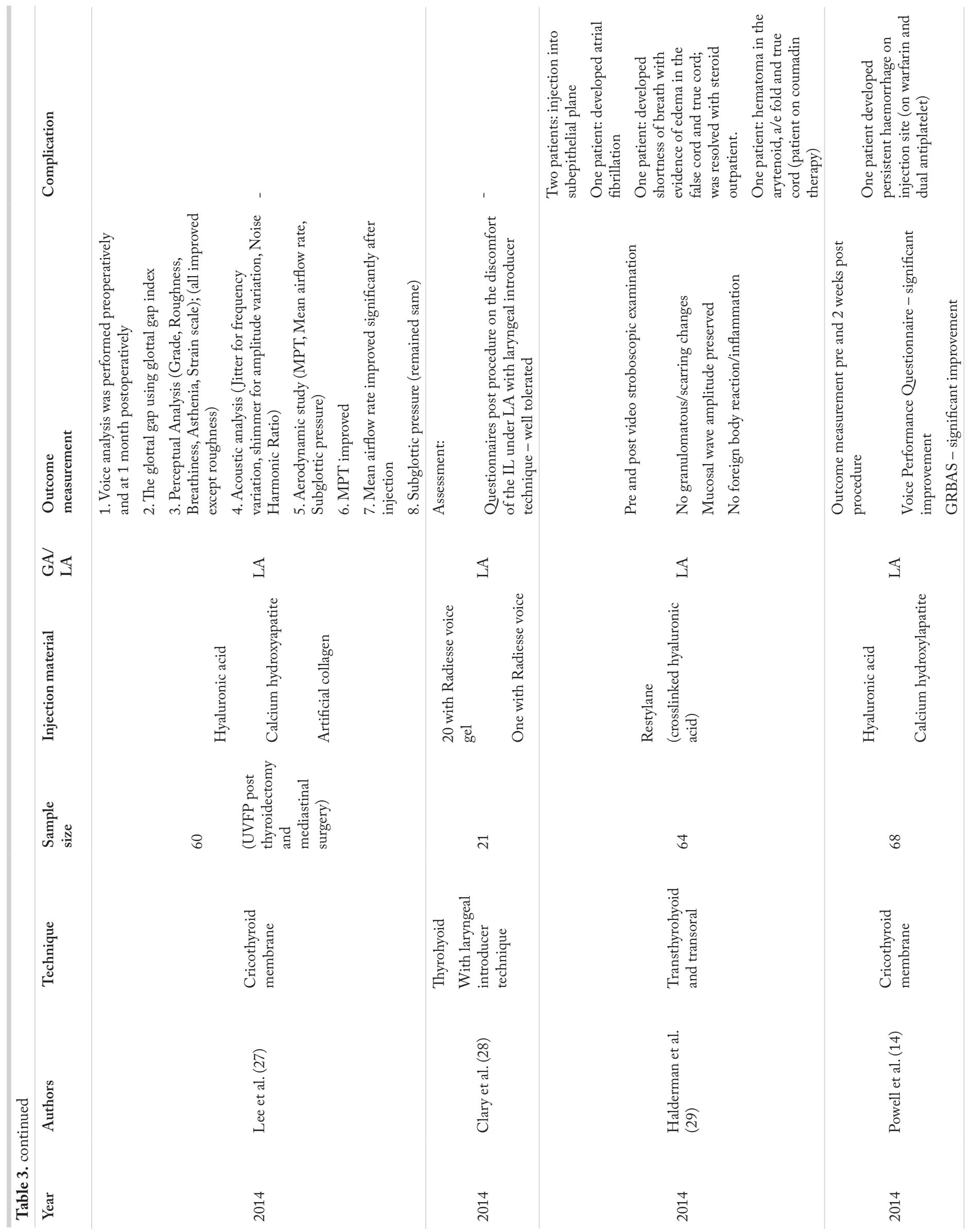




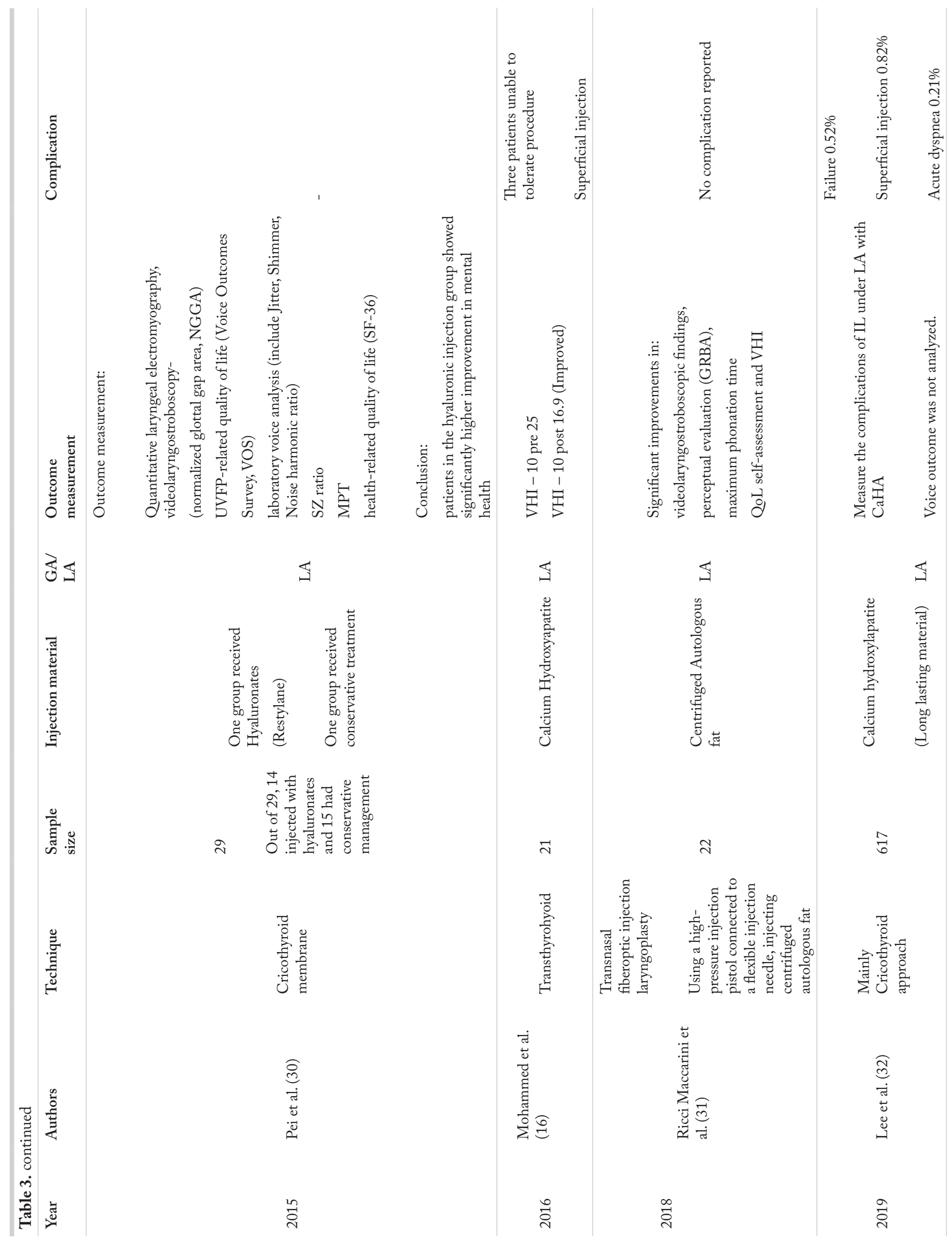


To accurately assess the voice outcome post injection laryngoplasty, a multidimensional holistic assessment is undoubtedly essential. To the best of the authors' knowledge, our research is among the very first few that have studied the multidimensional voice outcomes which consist of all subjective, objective, and acoustic analysis post early injection laryngoplasty under local anesthesia with the double-bend needle technique. According to our literature review, there are not many reported multidimensional voice outcome studies on double-bend needle technique injection laryngoplasty. Song et al. (6) reported voice outcomes with double-bend needle technique adopted mainly on subjective voice outcome survey and voice-related quality of life. Our multidimensional voice outcome study with the double-bend needle technique in post early injection laryngoplasty under local anesthesia showed significant improvement of the glottic function in the improvement of the objective and acoustic analysis, and no patients had any complications.

Hippocrates once said, "First, do no harm." Despite the promising multidimensional voice outcomes with reported low rates of complication in injection laryngoplasty under local anesthesia $(17,20)$, safe medical practice is crucial in modern medicine. We advocate injection laryngoplasty under local anesthesia as both a clinically and financially effective modality of treatment for patients with glottal insufficiency. However, it should only be done in a setting where the transfer of patients service and emergency equipment are available at all times should any complications arise (3). The procedure should be terminated if the patient is unable to tolerate it (17). Patients with pathological laryngeal condition, low pain threshold and those who require a concomitant operative procedure with the usage of direct laryngoscope and rigid telescope should avoid injection laryngoplasty under local anesthesia $(3,20)$. 
Proper glottic closure is important for generating cough and preventing aspiration during swallowing.

Multiple studies have shown that injection laryngoplasty benefits patients with glottic insufficiency in preventing aspiration (1). EAT-10 is a validated and symptom-specific outcome tool commonly used in clinical practice to measure swallowing difficulties. However, in our study, EAT-10 scoring was not included in view of the incomplete data recruited.

\section{Limitations}

Though the multidimensional voice outcomes post injection laryngoplasty under local anesthesia are promising, there are several limitations in our study.

1. We measured the multidimensional voice outcomes up to 3 months. A longer observational period would provide us with more concrete clinical evidence.

2. In all patients we used hyaluronic acid-based material with injection laryngoplasty; therefore, the comparative multidimensional voice outcomes of other injectable materials could not be evaluated.

3. Videolaryngostroboscopic assessments were not included in the presented study.

4. We adopted only the transthyrohyoid approach. Comparison of the outcomes with transthyroid and transcricothyroid approaches could not be made.

5. A study which includes EAT-10 would be more comprehensive, particularly in assessing the outcome of swallowing ability post injection laryngoplasty under local anesthesia.

The rationale of performing injection laryngoplasty under local anaesthesia as early as one day post recurrent laryngeal nerve (RLN) injury may be questionable as the neck is commonly edematous, inflamed and tender after thyroidectomy. Nevertheless, the injection procedure may be done under general anaesthesia or delayed until the neck is less swollen and tender. The senior author's experience in performing injection laryngoplasty in acutely injured RLN showed benefits to the patients especially in preventing patients from going home from the hospital with nasogastric feeding due to aspiration issues. Future studies also should investigate the safety and efficacy of injection laryngoplasty performed on-table or within few days of iatrogenic injury of RLN.

A future larger comprehensive study should be ideal to elucidate this information. Despite these considerations, the conclusion on the improvement of multidimensional voice outcome post injection laryngoplasty under local anesthesia from this database seems valid.

\section{Conclusion}

Early percutaneous transthyrohyoid injection laryngoplasty with the double-bend needle technique under local anesthesia is an effective modality for the treatment of glottic insufficiency with life and voice quality improvements. Hence, with the evidence of significant improvement of multidimensional voice outcome post early injection laryngoplasty, it should be highly considered in selected suitable patients with UVFP.

Ethics Committee Approval: Since this study is a retrospective study, ethics committee approval is not required.

Informed Consent: Retrospective study.

Conflicts of Interests: No potential conflict of interest relevant to this article was reported.

Financial Disclosure: The authors declared that this study has received no financial support.

\section{Main Points}

- Injection laryngoplasty under local anesthesia is a prudent and cost-effective treatment of unilateral vocal fold paralysis.

- The double-bend needle technique allows better visualization and access to the endolarynx resulting in a high success rate.

- Significant improvement on the multidimensional voice outcomes was evidenced by our series of subjective and objective evaluations.

\section{References}

1. Zuniga S, Ebersole B, Jamal N. Inpatient injection laryngoplasty for vocal fold immobility: When is it really necessary? Am J Otolaryngol - Head Neck Med Surg 2017; 38: 222-5. [Crossref]

2. Mathison CC, Villari CR, Klein AM, Johns MM. Comparison of outcomes and complications between awake and asleep injection laryngoplasty: A case-control study. Laryngoscope 2009; 119: 1417-23. [Crossref]

3. Bové MJ, Jabbour N, Krishna P, Flaherty K, Saul M, Wunar R, et al. Operating room versus office-based injection laryngoplasty: A comparative analysis of reimbursement. Laryngoscope 2007; 117: 226-30. [Crossref]

4. Ward PH, Hanson DG, Abemayor E. Transcutaneous Teflon injection of the paralyzed vocal cord: a new technique. Laryngoscope 1985; 95: 644-9. [Crossref]

5. Achkar J, Song P, Andrus J, Franco R. Double-bend needle modification for transthyrohyoid vocal fold injection. Laryngoscope 2012; 122: 865-7. [Crossref]

6. Song PC, Sung CK, Franco RA. Voice outcomes after endoscopic injection laryngoplasty with hyaluronic luronic acid stabilized gel. Laryngoscope 2010; 120(Supp14): S199-S199. [Crossref] 
7. Ong FM, Husna Nik Hassan NF, Azman M, Sani A, Mat Baki M. Validity and Reliability Study of Bahasa Malaysia Version of Voice Handicap Index-10. J Voice 2019; 33: 581.e17-581.e23. [Crossref]

8. Rosen CA, Lee AS, Osborne J, Zullo T, Murry T. Development and validation of the voice handicap index-10. Laryngoscope 2004; 114: 1549-56. [Crossref]

9. Mat Baki M, Wood G, Alston M, Ratcliffe, Sandhu G, Rubin JS, et al. Reliability of OperaVOX against Multidimensional Voice Program (MDVP). Clin Otolaryngol 2015; 40: 22-8. [Crossref]

10. Siau RTK, Goswamy J, Jones S, Khwaja S. Is OperaVOX a clinically useful tool for the assessment of voice in a general ENT clinic? BMC Ear, Nose Throat Disord 2017; 17: 1-6. [Crossref]

11. Teixeira JP, Oliveira C, Lopes C. Vocal Acoustic Analysis - Jitter, Shimmer and HNR Parameters. Procedia Technol 2013; 9: 111222. [Crossref]

12. Al-Yahya SN, Mohamed Akram MHH, Vijaya Kumar K, Mat Amin SNA, Abdul Malik NA, Mohd Zawawi NA, et al. Maximum Phonation Time Normative Values Among Malaysians and Its Relation to Body Mass Index. J Voice 2020; S08921997(20)30263-0. [Crossref]

13. Rosenthal LHS, Benninger MS, Deeb RH. Vocal fold immobility: A longitudinal analysis of etiology over 20 years. Laryngoscope 2007; 117: 1864-70. [Crossref]

14. Powell J, Carding P, Birdi R, Wilson JA. Injection laryngoplasty in the outpatient clinic under local anaesthetic: A case series of sixty-eight patients. Clin Otolaryngol 2014; 39: 224-7. [Crossref]

15. Mallur PS, Rosen CA. Vocal fold injection: Review of indications, techniques, and materials for augmentation. Clin Exp Otorhinolaryngol 2010; 3: 177-82. [Crossref]

16. Mohammed H, Masterson L, Gendy S, Nassif R. Outpatientbased injection laryngoplasty for the management of unilateral vocal fold paralysis - clinical outcomes from a UK centre. Clin Otolaryngol 2016; 41: 341-6. [Crossref]

17. Singh SK, Gupta SK. Vocal Fold Insufficiency: How Effective is Injection Laryngoplasty? Short Term Clinical Outcomes. Indian J Otolaryngol Head Neck Surg 2019; 71: 960-6. [Crossref]

18. Damrose EJ. Percutaneous injection laryngoplasty in the management of acute vocal fold paralysis. Laryngoscope 2010; 120: 1582-90. [Crossref]

19. Sulica L, Rosen CA, Postma GN, Simpson B, Amin M, Courey M, et al. Current practice in injection augmentation of the vocal folds: Indications, treatment principles, techniques, and complications. Laryngoscope 2010; 120: 319-25. [Crossref]

20. Alghonaim Y, Roskies M, Kost K, Young J. Evaluating the timing of injection laryngoplasty for vocal fold paralysis in an attempt To avoid future type 1 thyroplasty. J Otolaryngol - Head Neck Surg 2013; 42: 24. [Crossref]
21. 21. Yung KC, Likhterov I, Courey MS. Effect of temporary vocal fold injection medialization on the rate of permanent medialization laryngoplasty in unilateral vocal fold paralysis patients. Laryngoscope 2011; 121: 2191-4. [Crossref]

22. Choi N, Won S, Jin H, Kim HJ, Park W, Son YI. Additional Injection Laryngoplasty for Patients With Unilateral Vocal Fold Paralysis. Laryngoscope 2020; 130: 2863-8. [Crossref]

23. Wang C-T, Liao L-J, Huang T-W, Cheng P-W. Preliminary report of vocal fold augmentation with cross-linked porcine collagen. Otolaryngol neck Surg Off J Am Acad Otolaryngol Neck Surg 2012; 146: 606-10. [Crossref]

24. Birkent H, Sardesai M, Hu A, Merati AL. Prospective study of voice outcomes and patient tolerance of in-office percutaneous injection laryngoplasty. Laryngoscope 2013; 123: 1759-62. [Crossref]

25. Upton DC, Johnson M, Zelazny SK, Dailey SH. Prospective evaluation of office-based injection laryngoplasty with hyaluronic acid gel. Ann Otol Rhinol Laryngol 2013; 122: 541-6. [Crossref]

26. Wen M-H, Cheng P-W, Liao L-J, Chou H-W, Wang C-T. Treatment outcomes of injection laryngoplasty using cross-linked porcine collagen and hyaluronic acid. Otolaryngol neck Surg Off J Am Acad Otolaryngol Neck Surg 2013; 149: 900-6. [Crossref]

27. Lee YS, Kim YH, Kwon M, Ryu IS, Jung GE, Kim ST, et al. Shortterm treatment results for unilateral vocal fold palsy induced by mediastinal lesions. J Voice 2014; 28: 809-15. [Crossref]

28. Clary MS, Milam BM, Courey MS. Office-based vocal fold injection with the laryngeal introducer technique. Laryngoscope 2014; 124: 2114-7. [Crossref]

29. Halderman AA, Bryson PC, Benninger MS, Chota R. Safety and length of benefit of restylane for office-based injection medialization-a retrospective review of one institution's experience. J Voice 2014; 28: 631-5. [Crossref]

30. Pei YC, Fang TJ, Hsin LJ, Li HY, Wong AMK. Early hyaluronate injection improves quality of life but not neural recovery in unilateral vocal fold paralysis: An open-label randomized controlled study. Restor Neurol Neurosci 2015; 33: 121-30. [Crossref]

31. Ricci Maccarini A, Stacchini M, Mozzanica F, Schindler A, Basile E, De Rossi G, et al. Efficacy of trans-nasal fiberendoscopic injection laryngoplasty with centrifuged autologous fat in the treatment of glottic insufficiency due to unilateral vocal fold paralysis. Acta Otorhinolaryngol Ital 2018; 38: 204-13. [Crossref]

32. Lee M, Lee DY, Kwon TK. Safety of office-based percutaneous injection laryngoplasty with calcium hydroxylapatite. Laryngoscope 2019; 129: 2361-5. [Crossref]

33. Choi N, Jin H, Kim HJ, Son YI. Early injection laryngoplasty with a long-lasting material in patients with potentially recoverable unilateral vocal fold paralysis. Clin Exp Otorhinolaryngol 2019; 12: 427-32. [Crossref] 